\title{
ON THE MECHANISM OF GELATION IN REVERSI- BLE COLLOIDAL SYSTEMS
}

BY W. B. HARDY

Speaking generally, colloidal matter occurs in three conditions :

(I) As fluid mixture, colloidal solutions, or sols, as Graham called them;

(2) Solid mixtures of fluid and solid, the gels ; and

(3) Solids, such as dry silica or dry glass.

The property of forming gels is not possessed by all those mixtures which have been classed as colloids. Some only form slimes, which even to the point of actual drying retain the fluid property of flowing. Serum albumen and water is an instance.

Those which form gels fall into two well-defined classes, according to whether the change from the sol to the gel is, or is not, reversible by a reversal of the conditions which produce it. Silica and water may be taken as the type of the latter, gelatine and water of the former. When a hydrosol of silica forms a hydrogel, the latter is "insoluble." To this class belong hydrosols of metallic hydrosulphides and oxides. A hydrosol of gelatine sets to a hydrogel by lowering the temperature; the process is however reversed when the temperature is again raised. As the inner mechanism of the gelation of the hydrosols must differ in the two cases, since in the one irreversible, in the other reversible molecular aggregates are formed, I propose to distinguish the processes by different names. The production of an insoluble gel I will call "coagulation," of a soluble gel "setting." This nomenclature is in accordance with general usage.

Temperature is the most potent factor in determining whether a mixture which forms a reversible gel is in the sol or

\footnotetext{
${ }^{1}$ Read before the Royal Society, January 25, Igoo.
} 
gel state. There is also a limiting concentration of the solid below which the gel state is impossible at any temperature.

"Setting," as a rule, follows on a fall of temperature. Caseine, the chief proteid of milk, furnishes, I believe, the only known exception. In the presence of a small quantity of free alkali it forms a hydrosol. When a small quantity of a solution of calcium chloride or nitrate is added to this, a mixture is produced which forms a hydrogel on warming, and which reforms the hydrosol on again cooling. ${ }^{\mathrm{x}}$

\section{Part I. Reversible colloids}

Systems containing two or three components occur, that is, binary or ternary mixtures. The binary system, agar-and-water, was studied at considerable length; and initial experiments were made with the ternary systems, gelatine-water-alcohol, gelatinewater-mercuric chloride, and agar-water-alcohol. These mixtures are homogeneous when heated, but, on cooling, there occurs a division into two fluid phases. In the binary systems and in the ternary system agar-water-alcohol, the conjugate phases have approximately the same refractive index. In the ternary system, gelatine-water-alcohol, the refractive index of the one phase differs so much from that of the other as to permit of direct microscopical investigation of the form of the surface which separates the phases. For this reason I propose to treat this ternary system first.

The ternary system - gelatine-water-alcohol

When 13.5 grams of dry gelatine are dissolved in IOO $\mathrm{cc}$ of a mixture of equal volumes of absolute alcohol and water, a system is produced which is clear and homogeneous at temperatures above $20^{\circ}$. As the temperature falls below this limit a clouding occurs, which I find to be due to the appearance of fluid droplets which gradually increase in size until they measure $3 \mu$. On cooling further these fluid droplets become solid, and they begin to adhere to one another. In this way a framework is built up, composed of spherical masses hanging together in linear rows

I Sidney Ringer. Journal of Physiology, II, 464 (I890), 
which anastomose with one another. The framework therefore, is an open structure, which holds the fluid phase in its interstices. The macroscopical result of the change is the conversion, with falling temperature, of the fluid into a loose gel. The droplets can be readily separated from the interstitial fluid by the help of a centrifugal machine.

The phenomena above described undoubtedly depend upon the separation of a homogeneous mixture into two phases owing to a fall of temperature. Each phase contains water, alcohol, and gelatine, and the system may be described as a conjugate composed of a fluid solution of gelatine in a mixture of water and alcohol, and a solid solution of water with a trace of alcohol in gelatine. Both phases, however, are fluid within a small range of temperature. The surface of separation of the two phases is curved, and at first discontinuous, and owing to the small size of the droplets it is very large.

When the gel is heated the two phases again mix to form a clear fluid. Owing however to the fact that the droplets adhere to one another, they tend to fuse as temperature rises, so as to form irregular masses of viscous fluid, which are separated from the other phase by a surface of no particular shape. The irregular form of this surface, and the ease with which it is modified by any chance slight currents, show that at this stage the surface-tension between the two phases must be exceedingly slight. In order to simplify the description, I shall call that phase which separates as small spheres the "internal phase."

The concentration of the gelatine in the mixture exerts a very remarkable influence upon the configuration of the hydrogel. When it is present in large quantity the internal phase is less viscous and of smaller gelatine content than the external phase, and on cooling it is the external phase which becones a solid solution. The effect of increasing the proportion of gelatine above a certain amount is therefore very striking - it, so to speak, turns the system inside out, so that the gel is composed of a continuous framework of solid solution, out of which are hollowed spherical spaces filled with fluid. The general me- 
chanical properties of the gel, built on this plan, naturally differ very much from those of a gel with a small proportion of gelatine, which consists of an open framework of solid holding fluid in its interstices.

A mixture of gelatine, water, and alcohol is a ternary mixture which resembles a mixture of benzene, acetic acid, and water. In each there are two immiscible substances and a common solvent. The immiscible substances are gelatine and alcohol in the one case, and benzene and water in the other, while the common solvent is water in the former and acetic acid in the latter case. In both systems the solubility of the immiscible substances in the common solvent varies widely. Thus acetic acid and water, and water and alcohol, mix readily with rise of temperature; while acetic acid and benzene, and water and gelatine mix freely only when the temperature is above $15^{\circ}$ in the former case, and above $40^{\circ}$ in the latter case. Duclaux's researches ${ }^{x}$ show that in ternary mixtures having this last characteristic the distribution of the constituents in the two phases varies widely with variations in the composition of the whole mass.

These different characters are illustrated by the following figures, which give the amount of gelatine present in grams per roo cc. They are, however, only approximate for the solid phase, owing to the difficulty in separating it completely from the fluid phase.

Temperature $15^{\circ}$

\begin{tabular}{c|c|c}
\hline Total mixture & Internal phase & External phase \\
\hline 6.7 & I 7.0 & 2.0 \\
I 3.5 & 18.0 & 5.5 \\
36.5 & 8.5 & 40.0
\end{tabular}

The temperature at which the internal and external phases in this ternary system mix was found to be altered by altering the ratio of the masses of the components. Increasing the proportion of either of the two immiscible components, alcohol or

x Ann. Chim. Phys. (5) 7, 264 ( 1876 ). 
gelatine, was found to raise the temperature, while an increase in the proportion of the common solvent water was found to lower it.

The curvature of the surface which separates the phases was found not to be constant for a given mixture. The internal phase formed droplets which were large or small according to whether the mixture was cooled slowly or rapidly. Thus with a mixture containing I 3.5 grams gelatine per IOO cc the droplets (of solid solution) were very regularly $3^{\mu}$ in character when about $20 \mathrm{cc}$ was allowed to cool slowly in air. Cooled rapidly, however, in an ether spray, the droplets were so minute as barely to be visible with a magnification of 400 diameters. The effect of the rate of cooling is the same when mixtures with a large gelatine content are used, and when, therefore, the internal phase is a fluid solution at ordinary temperatures. When cooling is very rapid the droplets are excessively minute; when it is slow they may be as large as $10 \mu$ in diameter (gelatine 36.5 percent of the mixture). One can therefore make the general statement that the more slowly the division into two phases occurs the smaller and less curved is the surface of separation.

The effect upon the structure of the rate at which a fresh condition is imposed upon the system is manifested in a very striking way when an already formed gel is cooled. The experiments upon the effect of temperature on the composition of the two phases in the case of the hydrogel of agar show that when heat is added to or taken away from the system the balance of the phases is altered, water, and perhaps agar, passing from the one to the other. It might be expected that this would take place solely by the passage of material across the surface which separates the two phases. The study of the ternary mixtures, however, makes it clear that a new approximate equilibritum may be reached in two distinct ways.

When a portion of the hydrogel of gelatine-water-alcohol is cooled slowly from $I 6^{\circ}$ to, say $3^{\circ}$ or $4^{\circ}$, one can see with the microscope no change beyond an alteration in the size of the droplets already present, that is to say, the fresh (approximate) 
equilibrium is attained by exchange across the surface which separates the phases. But if the cooling is rapid, say a fall of $10^{\circ}$ in a few seconds, a secondary system of small droplets appears.

In all the mixtures which I examined these were formed in the external phase. Thus, when the concentration of gelatine in the whole mass was low, it was the fluid phase which underwent a division into secondary phases; when it was high, it was the solid phase. To put this fact in a general way, one can say that when the hydrogel is exposed to a rapid fall of temperature the phase which lies on the convex side of the surface of separation

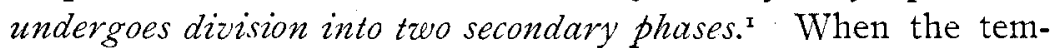
perature is again allowed to rise these secondary phases fuse before there is any obvious change in the relation of the primary phases.

When once formed the phases have considerable stability. If the droplets are composed of a solid solution one may, by the addition of water, cause them to increase to relatively vast dimensions without their being destroyed; as they increase in size their refractive index approximates more and more to that of the external phase until finally they are lost sight of. The addition of alcohol, however, once more brings them into view and causes them to shrink. Owing to this stability once a configuration is established one has to far overstep the conditions of its formation in order to destroy it. This would account for the remarkable hysteresis observed in reversible gels. Thus a ro percent solution of gelatine in water sets at $2 \mathrm{I}^{\circ}$ and melts again at $29.6^{\circ}$, and solutions of agar in water set at temperatures about $35^{\circ}$ and melt at temperatures about $90^{\circ}$. Similarly with the ternary mixtures. In one holding about 35 per cent gelatine, the internal and external phases separate at $20^{\circ}$, but they mix again only at $65^{\circ}$. When water is added to a ternary mixture so as considerably to swell the droplets the system is unstable, and the two phases mix at once when it is mechanically agitated.

1 The formation of the secondary phases therefore occurs in that one of the primary phases which is under the lower hydrostatic pressure. 
The properties of the ternary system : alcohol, gelatine, and water are the following:

I. Below a certain temperature it exists in two phases separated by a well-defined surface. The temperature at which the separation occurs depends upon the relative proportion of the components in the mixture. Increasing the proportion of gelatine raises it; as does also an increase in the proportion of alcohol. An increase in the proportion of the common solvent, water, however, lowers the temperature at which the biphasic character develops.

II. Both phases are at first fluid; with further fall in temperature one becomes solid.

III. The surface of separation is curved and discontinuous. In some cases, strictly as a secondary change, the discontinuous masses of the internal phase becomes continuous with one another.

IV. The more slowly the two phases are established the less is the surface which separates them, both in extent and in curvature.

$\mathrm{V}$. The solid solution phase is formed sometimes on the concave, sometimes on the convex side of the surface of separation. The former happens when the proportion of gelatine is small, the latter when it is large.

It follows from the last $(\mathrm{V})$ of these properties that a hydrogel may be built on two very different plans. It may consist of a solid mass containing spherical fuid droplets, or of solid droplets which, by hanging one to the other, form a framework in the spaces of which fluid is held. These two types present important mechanical peculiarities. The former is firm and elastic, and it maintains its structural integrity even under high pressure. The latter is much more brittle, and manifests a tendency to spontaneous shrinking, which is due to a continuous increase in the surface of contact or possibly union between droplet and droplet. These gels with an open solid framework therefore specially manifest that property of spontaneous shrinkage to which Graham applied the term "synæresis." 
In the building of a hydrogel of the second type two distinct events occur. The first is the separation of droplets, which rapidly become solid; the second is the linking of these droplets together to a pattern so that they build a framework throughout the fluid phase. The first is the separation of a homogenous mass into two phases; the second is a phenomenon akin to the grouping of particles which are suspended in a fluid. It is probable that these two events are not directly connected with one another.

\section{Binary mixtures (agar-water)}

I know of no binary reversible system in which the optical characters of the two phases differ sufficiently to permit of direct microscopical investigation of the surface of separation. It is, however, easy to prove that in such a system as agar and water the property of gel building is dependent upon the appearance of two phases.

The agar which was used was prepared from commercial agar as follows. The strips were suspended in a large volume of distilled water for twenty-four hours; the water was then drained off, and a large part of the water absorbed by the strips was squeezed out by a powerful press. The strips were again suspended in distilled water, and again drained and squeezed after forty-eight hours. This washing with distilled water was continued for some weeks. The strips were then melted and the hydrosol filtered, and the filtrate allowed to set. The clear hydrogel so obtained was sliced and suspended for a further period of weeks in many changes of distilled water. In this way a colourless gel was obtained free from all foreign diffusible bodies. It was not found necessary to take precautions against micro-organisms. With the removal of the salts the agarceased to afford them a suitable nidus.

\section{Effects of pressure upon the hydrogel of agar}

When gels containing I to 3 percent solids ${ }^{x}$ are broken up and slightly squeezed by hand a fluid exudes. In order to

\footnotetext{
' By this is meant I to 3 grams per 100 grams.
} 
collect this fluid a screw press was made use of. The gel was cut into pieces, which were wrapped in fine cotton canvas which had been completely freed from soluble substances by treatment for months with hot and cold distilled water. The packet was then pressed in a screw press, and the large yield of fluid collected. When the fluid ceased to flow, the solid which remained in the canvas was removed to a stoppered vessel.

The fluid was found to be a solution of agar. This was proved by evaporating some after it had been thrice filtered to a small bulk, when it was found to set to a typical clear gel. The results of the study of the ternary systems give sufficient grounds for defining the expressed fluid as a solution of agar in water, and the solid which remains in the canvas a solution of water in agar. The effect of the composition of the gel and of temperature upon the distribution of the water and the agar in these two phases was determined. The percentage composition was arrived at by drying a known weight of each, and assuming that the residue was entirely composed of agar. The results which were obtained lie far outside of any error which could have been introduced by this assumption when one considers the pains which were taken to free the gels from foreign bodies. Further, in every case an examination of the dry residue was made in order to prove that it was composed of matter capable of forming with water a typical agar gel.

\section{Experimental difficulties}

The method used to separate the two phases, thotigh at first sight crude, was found to be the most effective. The great error to be avoided is the blocking of the canvas pores by a mass of the solid phase, so that, instead of the true fluid phase, one really expresses fluid which has been forced through a membrane (pressure filtered). Owing to this error, a press, which I had specially made, and in which the piston drove the gel directly down on to a disc of canvas, proved quite useless. Very great force was necessary to express a fluid which was found to be almost pure water.

To succeed, it is necessary to avoid direct or great pressure. 
The masses of gel are loosely placed in a long canvas packet, which is then deformed by pressing the ends together. The pressure necessary to yield abundant fluid is now quite small, for the solid framework of the gel is destroyed by being rubbed against the canvas, and is reduced to fine particles, while the fluid easily makes its way through the coarse pores of the canvas. Raising the pressure always expresses a fluid poor in agar, while with slight to moderate pressure the concentration of the expressed fluid, as tested by determining the solids in the yield at different stages, remained fairly constant, but always with a slight decrease as time went on.

The expressed fluid was filtered before the solids were estimated; this was found to lower the amount of solid to a very slight extent.

The following figures illustrate the variation in the composition of the fluid as the gel becomes more completely expressed:-

Successive equal quantities of expressed fluid contain dry agar in Ioo cc. Temperature $14^{\circ}$

\begin{tabular}{c|c}
\hline Experiment I & Experiment II \\
\hline O.I2 & 0.I I \\
O.I4 & 0.12 \\
O.I & 0.09
\end{tabular}

The mechanical pressure used to separate the phases will modify their composition by deforming the surface of separation. This error cannot be estimated.

The influence of the ratio of the masses of the two components upon the composition of the phases

Two portions of a fairly concentrated gel were taken. To one part water was added to dilute it, and both were then heated to $100^{\circ}$, and equal portions of each were poured into two glassstoppered cylindrical vessels of identical shape, make, and size. The two vessels were then set aside to cool. 
After forty-eight hours samples were cut from different levels in each gel, and used to determine the percentage composition.

Five hundred and eighty grams of each of the gels were then expressed. The results were as follows:-

Temperature $18^{\circ}$

\begin{tabular}{|c|c|c|c|c|}
\hline \multirow{2}{*}{$\begin{array}{l}\text { Agar in } 100 \\
\text { grams of the gel }\end{array}$} & \multicolumn{2}{|c|}{ Expressed fluid } & \multicolumn{2}{|c|}{ Solid solution } \\
\hline & Volume & Agar & Volume & Agar \\
\hline grams & $\mathrm{cc}$ & percent & $\mathrm{cc}$ & percént \\
\hline I. I & 440 & 0.1 & 140 & 4.7 \\
\hline $3 \cdot 3$ & 230 & O.I4 & 350 & 5.6 \\
\hline
\end{tabular}

In another experiment :-

Temperature $15^{\circ}$

\begin{tabular}{r|l|l|l|l|l}
\hline .6 & - & 0.12 & - & - \\
2.2 & - & 0.14 & - & -
\end{tabular}

Thus an increase of the concentration of agar in the mixture produces an increase in the concentration of the agar in both phases. An explanation of this relation is suggested, and discussed later.

Effect of temperature upon the composition of the phases

This was determined by running a large mass of the hydrosol into a number of glass vessels of the same shape and size. Each vessel held $600 \mathrm{ce}$ of the hydrosol. They were close stoppered and allowed to cool to the room temperature. After fortyeight hours they were placed in chambers of known temperature, where they were kept for five to seven days before the contents were subjected to pressure. In these experiments, as is obvious, the internal changes are those which follow on raising or lowering the temperature of the hydrogel from the air temperature. In other experiments the hydrosol was cooled down to, but not below, the temperature of observation. This distinction is important, because it was found that the composition of the phases 
varied for a given temperature according to whether that temperature was the lowest of a descending series or the highest of an ascending series. This is shown clearly in the two curves $\mathrm{AB} \mathrm{AB}^{\prime}$, Fig. I. The arrows indicate the direction, ascending or descending, of the changes of temperature.

No. I.-Agar content of mixture I.6 percent

\begin{tabular}{|c|c|c|c|c|}
\hline & & Temperature & $\begin{array}{l}\text { Agar in } \\
\text { expressed fluid }\end{array}$ & Agar in solid \\
\hline \multirow[b]{2}{*}{ Ascending series } & & $14^{\circ}$ & 0.14 & 一 \\
\hline & & $\begin{array}{l}33 \\
50\end{array}$ & $\begin{array}{l}0.29 \\
0.80\end{array}$ & 二 \\
\hline \multirow[t]{2}{*}{ Descending series } & & 14 & O. 14 & 一 \\
\hline & 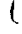 & 33 & I. IO & - \\
\hline
\end{tabular}

No. 2.--Agar content of mixture 2.23 percent

\begin{tabular}{|c|c|c|c|}
\hline & Temperature & $\begin{array}{c}\text { Agar in } \\
\text { expressed fluid }\end{array}$ & Agar in solid \\
\hline \multirow{3}{*}{ Ascending series } & $13^{\circ}$ & 0.12 & 4.7 \\
\hline & 36 & 0.25 & 5.0 \\
\hline & 5 & 0.09 & 3.0 \\
\hline \multirow{2}{*}{ Descending series } & $\begin{array}{l}13 \\
36\end{array}$ & 0.12 & 4.7 \\
\hline & $3^{\circ}$ & 0.47 & 3.2 \\
\hline
\end{tabular}

Putting on one side for a moment the different effect of an ascending or a descending temperature change, these experiments show that (I) a hydrogel of agar is a structure formed of a more solid part and a fluid, and (2) each of these two phases is a mixture of agar and water, (3) the composition of the phases is dependent to a lesser degree upon the ratio of agar to water in the entire mass, to a greater degree upon the temperature.

While recognizing as fully as possible that only an approximation to the actual composition of the two phases at different temperatures is obtained by these experiments, it is obvious that they afford reliable information on two points. These are, firstly, the marked "lagging" action or passive resistance to change offered by the system agar-water. The difference in composition of the phases according to whether any given temperature lies 
in an ascending or a descending series shows how slow the system is in reaching final equilibrium. ${ }^{\mathrm{I}}$ Secondly, the experimental results seem to me to indicate pretty clearly the general form of a part of the concentration temperature curve. I give the curve as it appears from the figures in Experiment II. AB and $C D$ are the curves for the system-solution of agar in water,

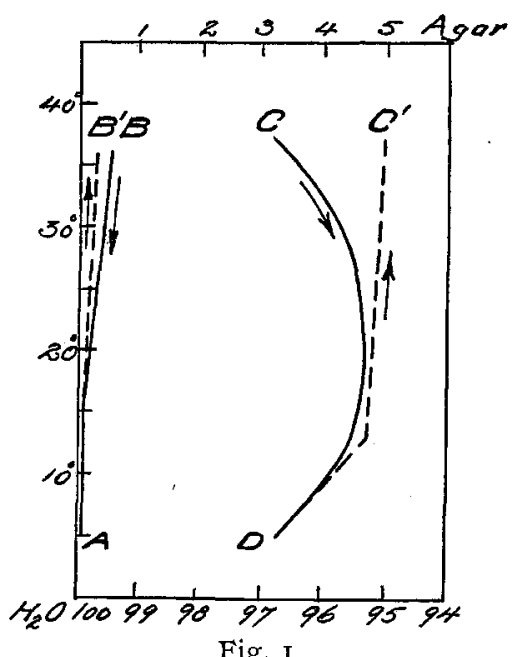

Fig. I

solution of water in agar, and vapor. If they correctly represent the general form of the curve, then, by the theorem of Le Chatelier, it follows that the change from the system solution of water in agar and vapor to the system solution of water in agar, solution of agar in water, and vapor will be accompanied by a liber-

${ }^{1}$ The system salicylic acid and water, and thorium sulphate and water are perhaps comparable cases. The former readily yields two fluid phases which. however, are throughout in labile equilibrium (Bancroft, 'The Phase Rule,' p. 105). In the case of the latter system supersaturated solutions can be obtained over a wide range of temperatures, and even in presence of the stable hydrates it is often hours or days before equilibrium is reached (Bancroft, loc. cit., p. 54, or Roozeboom, Zeit. phys. Chem, 5, 198 (189o). The lagging action in colloids which is so markedly shown by van Bemmelen's researches into the effect of time on the hydrogel of silicic acid, ceases to be extraordinary when one remembers that one of the phases is a solid. Gels reach equilibrium much more rapidly than does, for instance, a bar of metal in which the reaction velocity is so slow that final equilibrium may never be reached. 
ation of heat when the change takes place along the isotherms from $5^{\circ}$ to $\pm 20^{\circ}$, and by an absorption of heat when the change is along the isotherms $\pm 20^{\circ}$ to $35^{\circ}$, while the change from the system solution of agar in water and vapor to the system of two solutions and vapor will always be accompanied by absorption of heat.

I have not established this deduction experimentally, but it finds a considerable amount of support in the following facts. When water is allowed to dissolve in pure dry agar at $14^{\circ}$, a considerable amount of heat is given off. I cc of dry agar in coarse powder added to ro $\mathrm{cc}$ of water gives a rise of more than $6^{\circ},{ }^{1}$ while control experiments with carefully dried finest graphite or sand gave a rise of temperature of $\mathrm{O} . \mathrm{I} 5^{\circ}$ and $\mathrm{O} . \mathrm{I} 7^{\circ}$ respectively. Wiedemann and Liudeking ${ }^{2}$ also found that when dried gelatine absorbs water heat is liberated, but when gelatine saturated with water is dissolved in water heat is absorbed.

I have verified the general form of the curve $A B$ in a way which eliminates all the errors due to the expression of the fluid phase from the gel. A cylindrical column of gel $15 \mathrm{~cm}$ high was divided by two vertical cuts at right angles into four equal pieces. Four stoppered glass vessels were taken of the same size and shape, and in each, one of the pieces was placed and just covered with water. Two of the bottles were kept at $I 4^{\circ}$ for a week, and two at $44^{\circ}$; the water in both was found to have dissolved some of the agar, and to contain per Ioo grams of the solution 0.50 gram and 0.12 gram of dry agar respectively.

'The curves $A B, D C$ continued upward will meet at some point which marks the consolute temperature for agar and water. I have attempted to fix this point by observing the changes in the intensity of the beam of polarized light scattered normal to the ray when parallel light is passed through a gradually cooling hydrosol. The observations, which are still in an initial stage, have so far failed to fix the point.

I The mercury in the Beckmann thermometer was driven beyond the scale into the upper reservoir.

${ }^{2}$ Wied. Ann. 25, I45 (1885). 
The study of ternary systems under the microscope makes it probable that as the curves $A B, D C$ are continued upward they reach a point beyond which the equilibrium is no longer between a fluid solution and a solid solution, but between two fluid solutions.

The first worker to regard gelation as being due to the formation of two phases, one fluid and the other solid, was van Bemmelen. ${ }^{\mathrm{I}}$ He has given a suggestive discussion of the formation and structure of gels, based chiefly upon the manner in which amorphous material is precipitated from a solution, and he is led to the conclusion that "coagtulation or the precipitation of a gel from a solution seem to be similar phenomena; a desolution (Entmischung) which forms, not two layers completely separated from one another, but

"I. A framework of a material which is in a more or less transitional state between fluid and solid, and which presents those special properties to which the term colloid is applied.

"2. A fluid which is enclosed within this framework."

Van Bemmelen, however, does not consider that these two parts can be considered as two phases in the sense of the phase rule, since there is no sharp line between them, ${ }^{2}$ and he therefore concludes that the phase rule cannot be employed to elucidate the phenomena. This opinion is based upon a study of the equilibrium between the water content of various gels and the vapour-pressure, so patient and thorough as to give it very great weight. The curves of the equilibrium points are gradually bending lines if the dehydration of the gels is sufficiently slow, but if dehydration is relatively rapid there is a sudden change of direction (Fig. 2) when the water content is very much diminished ( $\mathrm{I}$ to $2 \mathrm{H}_{2} \mathrm{O}$ to $\mathrm{ISiO}_{2}$ ).

It is possible that the form of these curves does not necessarily depend upon the absence of a clear separation between the fluid and the solid portions of the gel. When one considers how small is the mutual solubility of silica and water and how slight

× Zeit. anorg. Chem. 18, 20 (I898).

${ }^{2}$ Ibid. I8, I2I ( 898 ). 
therefore the influence which a given mass of silica is likely to exert upon the vapour-pressure of even a relatively small mass of water, it is probable that the form of the curve is determined more by the operation of secondary influences, such as capillary

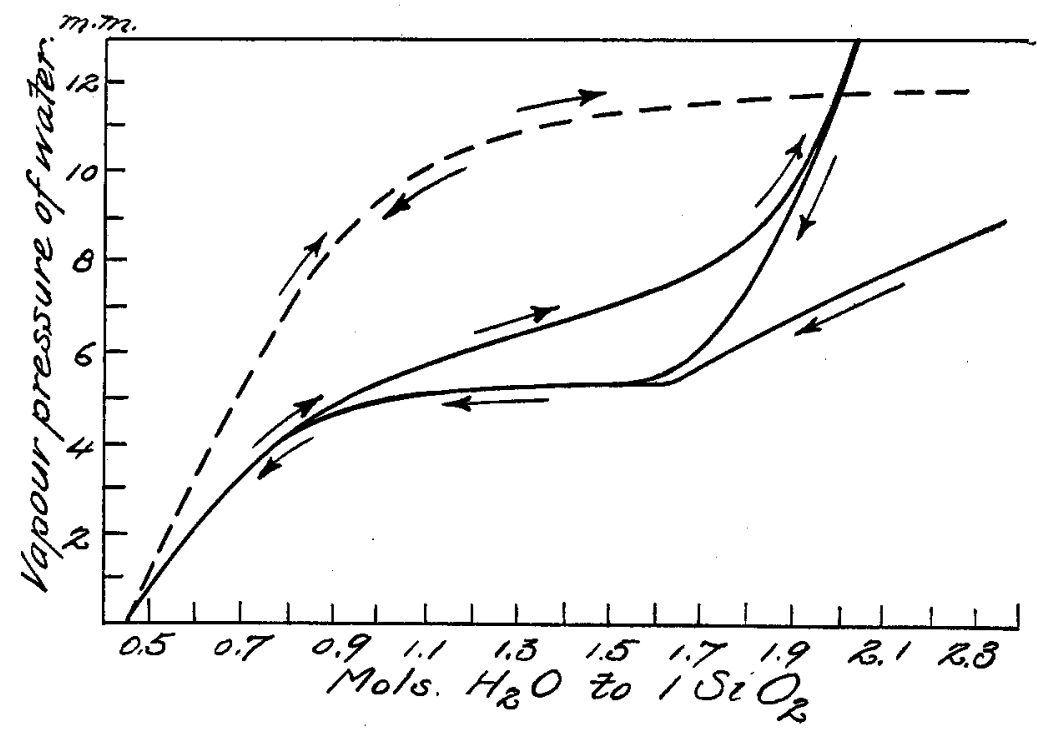

Fig. 2 (reproduced from van Bemmelen. Zeit. anorg. Chem. I3, 233 ( 1896$)$. - Equilibrium between a hydrogel of silica and water vapor.

Curve ..... the rate of removal of water very slow. In curve

much more rapid. The arrows indicate whether the curve shows the removal or the reabsorption of water.

tension, which depend on the structure of the gel, than upon the direct interaction of silica and water. Capillary tension would tend to lower the vapour-pressure ${ }^{x}$ with which the gel is in equilibrium to a greater and greater extent as the spaces in the solid framework of the gel became smaller and smaller with the decrease in the water content. The tendency to reduce the surface energy at the surface of separation of fluid and solid to a minimum, which manifests itself in the spontaneous shrinkage of some of these hydrogels, would act so as to raise the vapour-

1 The vapour-pressure which van Bemmelen measured is that of the free surface of the gel. It is analogous to that at the open ends of a number of capillary tubes filled with fluid. 
pressure with which the gel is in equilibrium, but the operation of this factor would diminish as the surface was diminished by decrease of the water content. These two forces operating simultaneously would alone produce the characteristic gradual diminution in the vapour-pressure of the gel as the fluid component is diminished. The break in the direction of the curve when dehydration has been relatively rapid and is nearly complete is what must occur when the capillary spaces in the framework become commensurate with the masses (small spheres for instance) of solid ont of which the framework is built, and when, therefore, any further diminution in the capillary spaces involves deformation of those masses, unless the removal of water is so slow that the very slow rate of readjustment in the solid phase is not exceeded. Lastly, the very limited powers of reabsorption of fluid by completely dried irreversible gels would, on this view, again not necessarily represent a reformation of the phases, that is to say, a real interaction between silica and water, but the refilling of capillary spaces by water due to the excessive capillary tension of these very minute capillaries. The capacity for reabsorption would therefore be diminished by any agent which facilitates the annealing of the dried gel and so destroys the capillary interspaces. Such an agent is heat, and van Bemmelen found that brief heating to red heat destroyed the reabsorptive powers of the gel of silica. ${ }^{\text {r }}$

There is one binary system in which gelation is an irreversible process (i. e. coagulation) which can be readily studied under the microscope. The hydrosol is a ternary system composed of water, a minute trace of free acid or alkali, and the modification of egg albumen which is produced by heating it to $100^{\circ}$. Coagulation occurs when the free acid or alkali is removed. As the coagulation point is neared the proteid particles in the hydrosol increase in size, so that spheres 0.75 to $\mathrm{I} \mu$ in diameter are formed. These become arranged in rows which anastomose so that an open net with regular polygonal meshes

г Zeit. anorg. Chem. ז3, 289 (I896). 
is formed. ${ }^{x}$ In this case the process of gel building is the same as that which can be followed so easily in ternary mixtures, and in both cases a definite surface separates the phases. It is probable that the hydrogel of silica is formed in the same way since Picton and Linder have shown by optical tests that, as the point of coagulation is approached, larger and larger particles of silica form in the hydrosol. ${ }^{2}$. These particles may be solid solutions of water in silica, or they may be large molecular aggregates of silica free from water. I incline to think that the latter is the more probable assumption since, if they were solutions, it is difficult to see why the process should be irreversible.

In the case of the reversible systems agar-water, or gelatinewater-alcohol, the particles seem to be of the nature of solid solutions.

The system agar and water consists of two components, and, therefore, a non-variant system should be defined by four coexistent phases. Since the gel stage consists of three phases, namely two solutions and a vapour phase, it should be a monovariant ${ }^{3}$ system. That is to say, the composition of the phases should be fixed by fixing either the temperature, or the pressure of the vapour phase. "The experiments show that this is not the case. The composition of the fluid and the solid phases is not constant for a given temperature. This result might be regarded as being due to the passive resistance to change in the system which is introduced by the formation of a solid phase. On this view, if the velocity of the reaction were known, the phases would be fixed if the element of time were introduced and accorded a definite finite value. This is the method which Bancroft suggests for dealing with such cases $;^{4}$ it is, however, possible that there are really more than $n+2$ independent variables

1 The process is described in detail in an earlier paper by the author in the Journal of Physiology, 24, I82 (I899), and the information which the microscope affords as to the manner in which irreversible gels are built is discussed there.

${ }^{2}$ Jour. Chem. Soc. 6I, I48 (1892).

${ }^{3}$ That is to say, a system having one degree of freedom.

${ }^{4}$ The Phase Rule, p. 234. 
so that the hydrogel is not a monovariant system. In an ordinary system the independent variables are the components $(n)$, temperature and pressure. Agar-water, however, is a system with two components, temperature and two pressures. This follows from the fact that the surface which separates the fluid and solid phases is curved. In point of fact the system is most closely represented by a system of two solutions separated by a membrane which is permeable by only one of the components, for while water will readily pass the surface of separation, agar, having the heavy immobile molecule characteristic of such organic bodies, will be almost unable to do so. Hence, if time be considered finite and small, the surface may practically be considered to be permeable by only one component. As Bancroft ${ }^{\mathrm{r}}$ points out, in a system of two solutions separated by semipermeable membrane there are two pressures and there will be $n+3$ phases in a non-variant system when $n=$ the number of components. The hydrogel is a system of three phases and, therefore, on this view, to fix the composition, it would be necessary to fix the temperature and one pressure. This relation would probably be true if the curvature of the surface of separation could be fixed. This, however, is not the case, and in order to fix the composition of the phases it would be necessary either to fix the temperature and both pressures, that of the internal as well as of the external phase; or to fix the temperature, one pressure, and the form of the surface. Practically we can only fix the temperature and the pressure of the external phase. I have succeeded in obtaining two phases separated by a plane surface by cooling a hydrosol slowly in an electric field. This method may prove suitable if the system is able to recover from the forces operating during its formation. The method of taking known weights of dry agar and water and keeping them at constant pressure and temperature until equilibrium is obtained is simple, but unfortunately there is the fallacy that the dry agar is a preformed system. The structure of the hydrogel from which it is reproduced is not destroyed by drying, and the sys-

I loc. cit. 
tem tends to reform itself on the old lines by the filling of the original capillary spaces.

To sum up these remarks we may describe the hydrogel of agar as a system of three phases, a solid, a fluid, and a vapour phase. The equilibrium is determined by the chemical potential of the components in the various phases, by two pressures, and by temperature. Other operating variables are capillary tension and the energy of the surface between the fluid and solid phases. I have made no measurements to determine how soon the system reaches equilibrium, but the analogous system, gelatine and water, attains to a constant melting-point twenty-four hours after the formation of the hydrogel. ${ }^{x}$

${ }^{1}$ Gelatinöse Lösungen, van der Heide, München, I897. 